REVIEW

\title{
Biomechanics and tennis
}

\section{B Elliott}

Br J Sports Med 2006;40:392-396. doi: 10.1136/bjsm.2005.023150

Success in tennis requires a mix of player talent, good coaching, appropriate equipment, and an understanding of those aspects of sport science pertinent to the game. This paper outlines the role that biomechanics plays in player development from sport science and sport medicine perspectives. Biomechanics is a key area in player development because all strokes have a fundamental mechanical structure and sports injuries primarily have a mechanical cause.

Correspondence to: Professor Elliott, University of Western Australia Perth, WA, Australia; bruce.elliott@uwa.edu.au

Accepted 10 December 2005
$\mathrm{S}$ uccess in tennis is greatly affected by the technique a player uses and biomechanics plays an integral role in stroke production. All strokes have a fundamental mechanical structure, and sports injuries primarily have a mechanical cause. ${ }^{1}$ Player development based on scientific evidence allows an individualised approach to be structured, with due consideration to the key mechanical features of each skill, while also fostering flair and permitting the physical characteristics of a player to be considered. An understanding of biomechanics from a sports medicine perspective is also important if player development is to occur with minimal risk of injury.

The examples given in the following sections are intended to reflect general directions rather than provide a comprehensive review of the literature. More detailed reviews can be found in the ITF publication Biomechanics of advanced tennis, ${ }^{2}$ and the books From breakpoint to advantage, $^{3}$ The physics and technology of tennis ${ }^{4}$ and Biomechanical principles of tennis technique: using science to improve your strokes. ${ }^{5}$

The review will be presented under a number of headings that reflect the different areas in which biomechanics is important to player development. Firstly, biomechanics from a general perspective will be followed by the role it plays in stroke production. Sports medicine, as it plays a role in the development of stroke production, is then discussed from a biomechanical perspective.

\section{GENERAL THEORY OF BIOMECHANICS}

Biomechanics theory provides coaches, players, and sport science support staff with a general framework for the development of stroke production. I will discuss a number of the general principles that guide this development.

\section{Muscle pre-tension (elastic energy)}

In a stretch-shorten cycle, elastic energy stored during the eccentric phase of the action (the stretch) is partially recovered, such that the concentric phase (shorten) is enhanced. This is also supported by the fact that the concentric action begins with the appropriate muscles under higher tension than would be created if they were to contract purely concentrically from a resting state. Research has shown that the benefit to performance from these two factors, particularly the muscle pre-tension, is critical to success in sports such as tennis. ${ }^{6}$ Examples from selected strokes are:

- Service: A subtle coaching point in maximising power in the serve is the timing of the "leg drive" with the racquet preparation for the drive to the ball. The eccentric stretch and pretensing of the anterior shoulder muscles (particularly the internal rotators) is maximised by a vigorous leg drive which positions the racquet "down behind and away from the lower back" in preparation for the drive to the ball.

- Groundstrokes: Rotation of the shoulders greater than the hips (creating a separation angle) and the positioning of the upper limb relative to the trunk during the backswing phase of these strokes, place appropriate muscles on stretch. This is why in the backhand a separation angle (one handed $\sim 30^{\circ}$; two handed $\sim 20^{\circ}$ ) is created in the backswing in preparation for the swing to the ball. ${ }^{7}$

- Volley/service return: The split step, an integral part of preparation for a volley, service return, or groundstroke, places the quadriceps muscle (extensor at the knee joint) on stretch, permitting storage and subsequent release of energy to enhance quick movement in preparation for the subsequent stroke.

The key to the recovery of the elastic energy is the timing between the stretch and shorten phases of the motion. The benefit of this stored energy is reduced if a delay occurs between these phases of the movement. In the bench press, after a period of about one second, $55 \%$ of the stored energy was lost. ${ }^{8}$ Elliott et al ${ }^{9}$ showed that speed of internal rotation of the upper arm was increased by about $20 \%$ for a no-pause compared with a 1.5 second pause condition. In tennis it is therefore essential that only a short pause occurs between the backswing and forwardswing phases of stroke production or at maximum knee flexion during the serve.

\section{Kinetic chain}

There are generally two segment coordination strategies used in tennis (table 1). In strokes where power is required (such as the service and groundstrokes), a number of body segments must be coordinated in such a way that a high 
Table 1 Coordination strategies in tennis

\begin{tabular}{|c|c|c|}
\hline \multicolumn{2}{|l|}{ Power stroke (the service) ${ }^{10}$} & Precision stroke (the volley at net)" \\
\hline \multicolumn{2}{|c|}{$\begin{array}{l}\text { Leg drive and trunk rotations } \Rightarrow \text { shoulder speed } \\
\text { (forward/shoulder-over- } \\
\text { shoulder/twist) }\end{array}$} & $\begin{array}{l}\text { Shoulder rotation, forward step of } \Rightarrow \text { racquet speed } \\
\text { the front leg, and forward } \\
\text { movement of the racquet arm all } \\
\text { work together as a unit }\end{array}$ \\
\hline \multicolumn{3}{|c|}{+} \\
\hline \multicolumn{3}{|c|}{$\begin{array}{l}\text { Upper arm elevation and } \Rightarrow \text { elbow speed } \\
\text { flexion }\end{array}$} \\
\hline \multicolumn{3}{|l|}{+} \\
\hline \multicolumn{3}{|l|}{$\begin{array}{l}\text { Forearm extension and } \\
\text { pronation and upper arm } \\
\text { internal rotation }\end{array}$} \\
\hline$\stackrel{+}{\text { Hand flexion }}$ & $\Rightarrow$ racquet speed & \\
\hline
\end{tabular}

racquet speed is generated at impact. Where precision is needed, the number of segments is reduced and segments operate more as a unit (such as the volley at the net), although the drive volley now challenges this general principle.

Efficient function, with maximal performance and minimal risk of injury, requires optimum activation of all the links in the kinetic chain designed for power. ${ }^{12}$ Injury is often associated with alterations in the flow of energy across segments, such that if one segment is removed from the chain, then there is an increased reliance on the others to accommodate this loss, which may lead to tissue overload.

\section{Variability in stroke production}

Work by Knudson ${ }^{13}$ has shown that selected aspects of the stroke production of high performance players are variable, whereas other aspects are repeatable. For instance, in the forehand drive, wrist and elbow angular positions are generally consistent at impact. However, this repeatable angular position was not the result of highly consistent patterns of angular velocity and acceleration of the same joints. That is, a given end result is achieved through a variety of movement strategies. Coaches in developing stroke production must then vary drills-for example, pace, spin, direction, and height of ball feed or drill structure-to develop a variety of neuromotor timings associated with a given stroke, to achieve the almost infinite combinations of racquet speed, trajectory, and impact characteristics associated with a successful return.

\section{Analysis protocols}

Technique analysis falls on a continuum between subjective (qualitative) and more objective (quantitative) analyses. Research shows that an expansive view of subjective technique analysis, the procedure generally used by coaches, is required if performance is to be optimised. Any effective process must evaluate movement to identify both strengths and weaknesses in performance and then diagnose the movement to prescribe an appropriate intervention. ${ }^{15}$

A variety of models of technique analysis have been proposed. However, they may generally be divided into approaches that focus on:

- analysing general biomechanical principles-for example, assessment of balance during stroke production, followed by the application of force and inertia in the development of racquet speed and so on

- identifying variables that are structured in a deterministic model-the mechanical method (see fig 1 for a model of the serve). The advantage of this approach is that the relations between variables is highlighted

- key technique points (position of the racquet at the completion of the backswing or impact) being identified
The complexity of fig 1 is one reason why coaches often prefer to use the "critical points" approach as the basis for their analyses.

Irrespective of what procedure is used, the four stage approach to technique development (preparation, observation, intervention, and reassessment) developed by biomechanists in combination with pedagogists has had a major influence on the way tennis stroke production is analysed, interventions developed, and performance reassessed.

\section{Equipment design}

A discussion of this topic is beyond the scope of this paper, and the reader is directed to the book by Brody et $a l^{4}$ for a complete review of this broad topic. However, there is no doubt that modern racquets have enabled the ball to be hit with a higher speed than was possible with previous designs. Lighter racquets with larger "areas of percussion" and new string designs have all affected modern technique and are the primary reason for a number of changes to stroke production discussed below.

\section{BIOMECHANICS OF STROKE PRODUCTION Serve/groundstrokes}

Internal rotation of the upper arm at the shoulder

Work by my team has primarily been responsible for identifying the important role that internal rotation of the upper arm at the shoulder joint plays in the service (fig 2) and the forehand strokes (table 2). ${ }^{10}{ }^{14}$ This factor has in many ways modified the way that these strokes are developed at beginner and advanced levels of play. The images in fig 2 show how internal rotation at the shoulder, which begins before impact, continues into the early follow through phase of the service action. Functionally, the internal rotator musculature must accelerate the upper arm in the swing to impact, before the external rotators eccentrically contract to decelerate this rotation during the follow through phase of the action. As the external rotators are much smaller than their internal rotator counterparts, it is essential that specific training is structured to protect the shoulder from injury, as discussed in the sports medicine section below.

Remember, these figures relate to the approximate contributions at impact and take no consideration of such movements as leg drive in the service action. They are indicators of the movements responsible for the generation of racquet speed and do not indicate the importance of a particular movement in a stroke. For instance, in the power serve, pronation is primarily responsible for racquet orientation, and elbow extension assists in the generation of impact height.

Trunk rotations in the serve

The view most commonly held by coaches was that a player rotated the trunk horizontally about a near vertical axis 


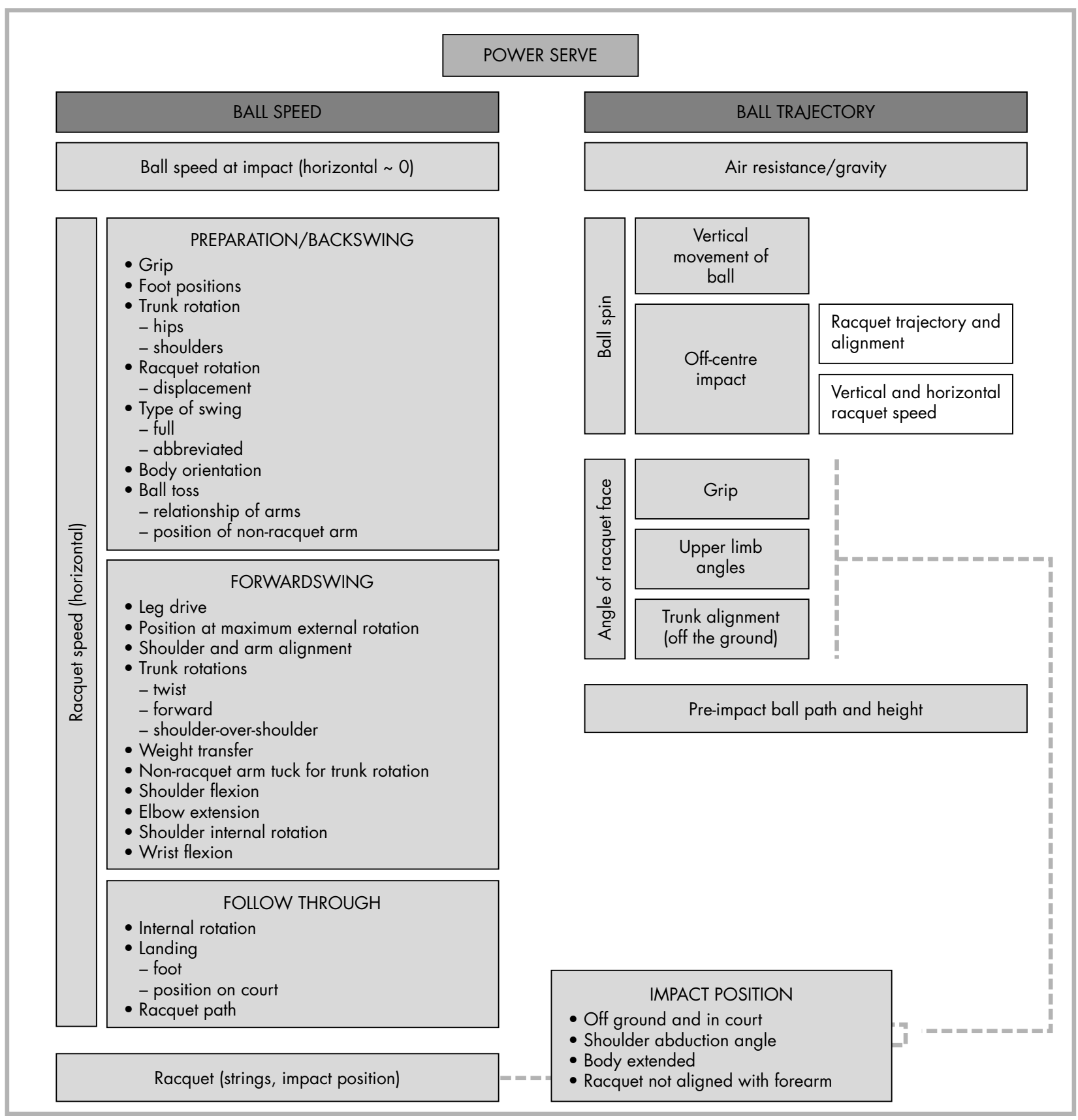

Figure 1 Model of the power serve in tennis. Published with permission of the International Tennis Federation.

during the forward swing in the service action. Players knew better and have tilted the trunk to rotate more in a shoulderover-shoulder orientation, rather than a simple horizontal rotation of the shoulders. They obviously positioned themselves in this manner to enable internal rotation of the upper arm at the shoulder to play the important role in the service action that we now know it does (fig 2). Research by Bahamonde ${ }^{16}$ on angular momentum in the trunk during the serve clearly showed the importance of shoulder-overshoulder and forward rotation movements during the service action. Although science again cannot claim that it was the reason for these movements, it certainly played an integral role in their integration into player development. Current research by Elliott, Sweeney, Alderson, and Reid is aimed at quantifying the role of the "back leg drive" in the generation of shoulder-over-shoulder trunk rotation in the service action.
Lower limb and pelvic drive in groundstrokes

Research at the University of Tokyo has provided insight into the role of lower limb drive and pelvic rotation in the forehand ${ }^{17}$ and backhand ${ }^{18}$ strokes. The importance of internal extensor moments at the back hip was identified in both the above studies. Again the importance of hip (pelvic) rotation has been highlighted in both forehand and backhand strokes.

\section{SPORTS MEDICINE}

\section{Loading}

Epidemiological data have shown that tennis injuries are primarily caused by overuse. ${ }^{3}$ Loading (the rate of force development, peak force, and torque are mechanical factors that collectively are often referred to as "load") may be applied to the body externally (ground reaction force, 

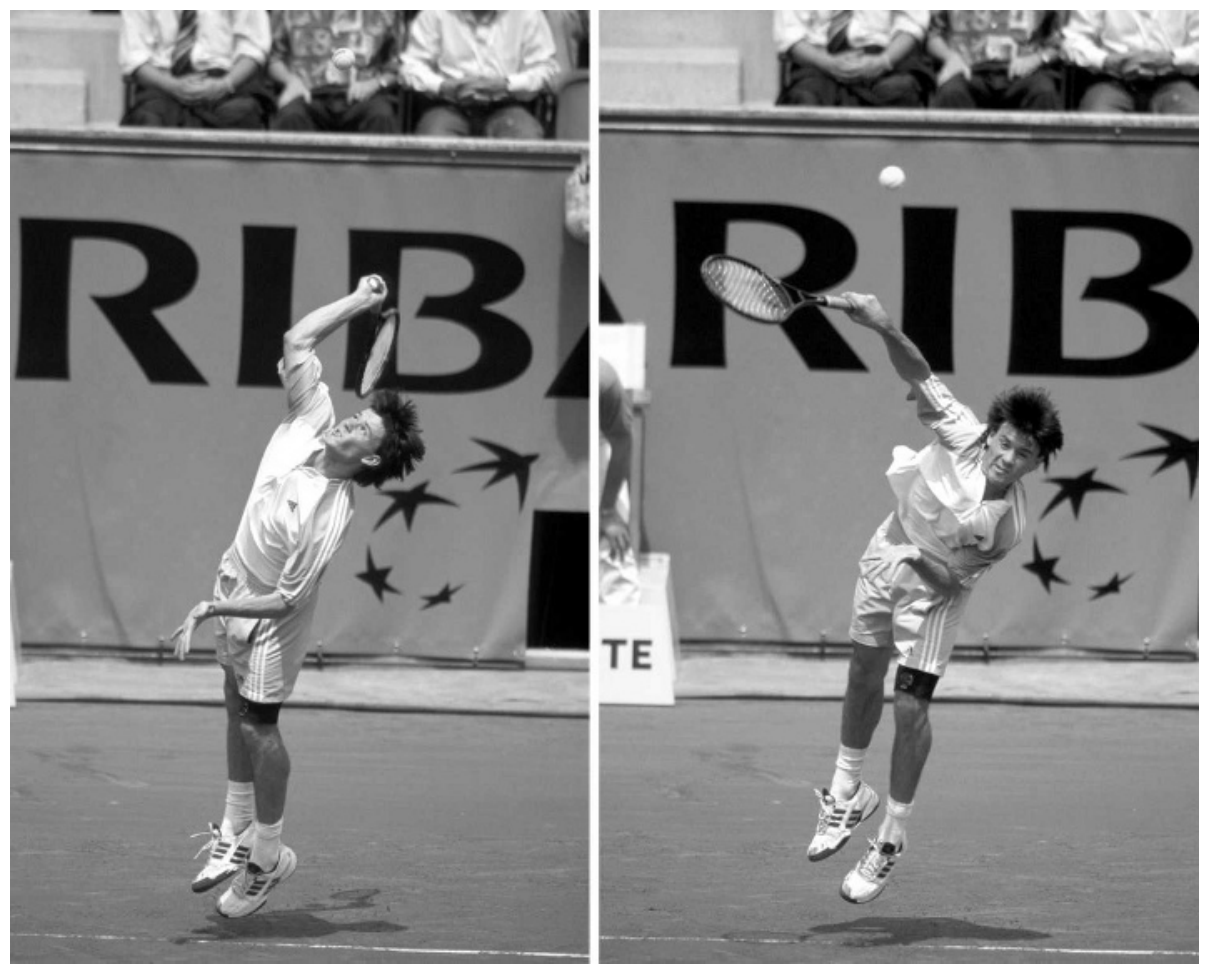

Figure 2 Pictures of service action showing shoulder-over-shoulder trunk rotation and internal rotation of upper arm at the shoulder joint. Published with permission of the International Tennis Federation. The player has given permission for publication of this figure.

vibration) or internally (muscle forces and torques). Research on elite players at the Sydney Olympics provided an insight into the load placed on the shoulder and the elbow joint of these players. ${ }^{19}$ These data provided information on leg drive, the abbreviated backswing, and service speed as they affected shoulder and elbow loading. In very general terms, this paper showed the following.

- Loading increased with an increase in service speed. That is, irrespective of sex, if a player wishes to develop a more powerful serve they need to modify technique (rotate selected segments more quickly) and prepare the body physically to perform the higher speed movement(s).

- Those players with more effective knee flexion-extension during the service action were associated with lower loading at the shoulder and elbow. This finding needs to be further clarified such that the effect of leg drive on loading is assessed; this is currently taking place at the University of Western Australia.

- There was a trend for players with very abbreviated backswings to record higher force levels at the shoulder. This finding is also currently being tested in a more controlled design.

Staying on the topic of loading and the tennis serve, Chow et $a^{20}$ investigated activation on the lower trunk muscles during various types of service actions. They showed that coactivation of lower trunk muscles assists in the stabilisation of the lumbar spine during extension/flexion and rotation of this region during the service action. The abdominal muscles were more active in the topspin serve than the flat and slice serves during the upward swing of the racquet to impact. These results reinforced the importance of abdominal and low back exercises (core stability) together with the muscles about the shoulder and lower limbs in strength and rehabilitation programmes in tennis.

\section{Tennis elbow}

The prevalence of lateral humeral epicondylitis is acknowledged as a condition that primarily occurs in the recreational athlete. Research on the one handed backhand stroke shows that this condition may be related to the use of faulty stroke mechanics by novice players. ${ }^{21}$ Skilled players impact the ball with a hyper-extended wrist and extend the hand through impact. That is the extensor muscles about the wrist joint act concentrically to develop racquet speed through impact. However, novice players often strike the ball with the wrist flexed $\left(\sim 13^{\circ}\right)$, while moving the hand at the wrist joint into further flexion. That is the extensor muscles about the wrist joint contract eccentrically, before the contraction concentrically following impact. Wrist extensor electromyography for both groups showed similar levels of activity in the period before ball impact, whereas the skilled players showed greater electromyographic levels after contact.

The above study was followed by another with a similar design but with professionals and intermediate level players

Table 2 Approximate contributions to impact racquet velocity (\%)

\begin{tabular}{lll}
\hline & Power serve & $\begin{array}{l}\text { Topspin forehand } \\
\text { (forward direction) }\end{array}$ \\
\hline $\begin{array}{l}\text { Shoulder } \\
\text { Upper arm } \\
\quad \text { Horizontal flexion } \\
\quad \text { Internal rotation }\end{array}$ & 10 & 15 \\
$\begin{array}{l}\text { Forearm } \\
\quad \text { Extension }\end{array}$ & 15 & 25 \\
$\quad \begin{array}{l}\text { Pronation } \\
\text { Hand } \\
\quad \text { Flexion (palm/ulnar) }\end{array}$ & Negligible & Negligible \\
$\quad$ Flexion (palmar/radial) & 50 & Negligible \\
\hline
\end{tabular}


with and without a history of tennis elbow. ${ }^{22}$ Hand angular velocity at the wrist, measured by an electrogoniometer, was significantly different in the professionals $(4 \mathrm{rad} / \mathrm{s}$, extension) and intermediate group who had a history of tennis elbow (0.4 rad/s, flexion). As with the study by Blackwell and Cole, ${ }^{21}$ lateral epicondylitis has been associated with an eccentric contraction of the wrist extensors at impact. No significant differences were found in impact acceleration or elbow kinematics between the three groups.

\section{Muscle balance}

One of the possible causes of shoulder injury may be peak strength/torque imbalances in those muscles responsible for acceleration and then deceleration of the upper arm at the shoulder joint. Certainly work by Tod Ellenbecker and Paul Roetert has provided players, coaches, and sports medicine personnel with isokinetic profiles of highly skilled and junior tennis players. ${ }^{23}$ The comparison of the concentric strength of one muscle group compared with the eccentric strength of the antagonist group in throwers was explored by Noffal. ${ }^{24} \mathrm{He}$ concluded that rehabilitation and injury prevention regimens that include exercises for eccentric external rotation strength may bring more balance to the dominant shoulder of throwing athletes. Such information is critical to the preparation of athletes where explosive concentric and eccentric contractions are common place. Remember, in most tennis strokes, the stretch-shorten cycle is characterised by eccentric followed by concentric contractions. For example in the serve you have:

- external upper arm rotation at the shoulder during the backswing slowed by eccentric contraction of the internal rotators at the shoulder

- concentric contraction of the shoulder internal rotators, in the drive to the ball, is then slowed by an eccentric contraction of the shoulder external rotators during the follow through

It is therefore apparent that we must train muscles in concentric and eccentric modes, while also recognising that large muscle imbalances will lead to injury. This is particularly true in the eccentric contraction during the follow through, as the external rotators at the shoulder are required to "decelerate" the rapidly internally rotating upper arm. Prehabiliation or exercises to minimise loading stresses should be included in any training programme before a return to full training. ${ }^{25}$

\section{CONCLUSIONS}

There is no question that players striving for more power, more control, or more variety in stroke production through trial and error are the primary determinants in changes to stroke mechanics. However, I have shown that biomechanics certainly plays a role in the process of change. General theory provides a base on which modifications can be made, and an understanding of individual stroke mechanics inevitably leads to improved performance. Science also enables players to modify their training regimens with minimal risk of injury. I have no doubt that the other sports science disciplines (psychology, exercise physiology, and pedagogy/motor learning) could also make a case for their role in player development.

Areas where science can play an improved role are in the linking of growth and development of the body, pedagogy, and skill development. When should selected biomechanical principles be introduced? When should we teach the leg drive in the service action from skill development and lower limb strength perspectives? From a tissue loading perspective, should we stress the importance of energy storage and muscle pre-tension at an early age? Although sport science
What is already known on this topic

- The biomechanics of tennis has been well researched, but publications are spread over a wide range of journals

- Furthermore, interpretations of findings are in various formats, thus limiting their application

What this study adds

- This review succinctly integrates biomechanical research from performance and sports medicine perspectives

has certainly assisted tennis development, it is also important to realise that much is still to be accomplished.

Competing interests: none declared

The player in figure 2 has given permission for its publication.

\section{REFERENCES}

1 Whiting W, Zernicke R. Biomechanics of musculoskeletal injury. Champaign, IL: Human Kinetics, 1998

2 Elliott B, Reid M, Crespo M, eds. Biomechanics of advanced tennis. London: International Tennis Federation, 2003.

3 Pluim B, Safran M. From breakpoint to advantage. Solana Beach, CA: Racquet Tech Publishing, 2004.

4 Brody H, Cross R, Lindsay C. The physics and technology of tennis. Solana Beach, CA: Racquet Tech Publishing, 2002

5 Knudson D. Biomechanical principles of tennis technique: using science to improve your strokes. Solana Beach, CA: Racquet Tech Publishing, 2006.

6 Walshe A, Wison G, Ettema G. Stretch-shorten cycle compared with isometric preload: contributions to enhanced muscular performance. J Appl Physiol 1998;89:97-106.

7 Reid M, Elliott B. The one- and two-handed backhands in tennis. Sports Biomech 2002; 1:47-68.

8 Wilson GJ, Elliott B, Wood G. The effect on performance of imposing a delay during a stretch-shorten cycle movement. Med Sci Sports Exerc 1991;23:364-70.

9 Elliott B, Baxter K, Besier T. Internal rotation of the upper arm segment during a stretch-shorten cycle movement. J Appl Biomech 1999:15:381-95.

10 Elliott BC, Marshall RN, Noffal G. Contributions of upper limb segment rotations during the power serve in tennis. J Appl Biomech 1995; 11:433-42.

11 Elliott BC, Overheu P, Marsh P. The service line and net volleys in tennis: a cinematographic analysis. J Sci Med Sport 1988;20:10-18.

12 Kibler B. Kinetic Chain contributions to elbow function and dysfunction in sports. Clin Sports Med 2004;23:545-52.

13 Knudson D. Intra-subject variability of upper extremity angular kinematics in the tennis forehand drive. International Journal of Sport Biomechanics 1990;6:415-21.

14 Elliott B, Takahashi K, Noffal G. The influence of grip position on upper limb contributions to racket head velocity in a tennis forehand. J Appl Biomech 1997; 13:182-96.

15 Knudson D, Morrison. Qualitative analysis of human movement, 2nd ed. Champaign, IL: Human Kinetics, 2002.

16 Bahamonde R. Changes in angular momentum during the tennis serve. J Sports Sci 2002;18:579-92.

17 lino $Y$, Kojima T. Torque acting on the pelvis about its superior-inferior axis through the hip joints during a tennis forehand stroke. Journal of Human Movement Studies 2001;40:269-90.

18 Akutagawa S, Kojima T. Trunk rotation torques through the hip joints during the one- and two-handed backhand tennis strokes. I Sports Sci 2005;23:781-93.

19 Elliott B, Fleisig G, Nicholls R, et al. Technique effects on upper limb loading in the tennis serve. J Sci Med Sport 2003;6:76-87.

20 Chow J, Shim J-h, Lim Y-t. Lower trunk muscle activity during the tennis serve. J Sci Med Sport 2003;6:512-18.

21 Blackwell J, Cole K. Wrist kinematics differ in expert and novice tennis players performing the backhand stroke: Implications for tennis elbow. J Biomech 1994;27:509-16.

22 Knudson D, Blackwell J. Upper extremity angular kinematics of the onehanded backhand drive in tennis players with and without tennis elbow. Int J Sports Med 1997; 18:79-82.

23 Ellenbecker $T$, Roetert $P$. An isokinetic profile of the trunk rotation strength in elite tennis players. Med Sci Sport Exerc 2004;36:1959-63.

24 Noffal G. Isokinetic eccentric-to-concentric strength ratios of the shoulder rotator muscles in throwers and non-throwers. Am J Sports Med 2003;31:537-41.

25 Kibler B. Rehabilitation of rotator cuff tendinopathy. Clin Sports Med 2003;22:837-47. 